\title{
LETTER
}

\section{Effect of virtual information on the satisfaction for decision-making among family members of critically ill COVID-19 patients}

\author{
Juliano Ramos ${ }^{1}$, Caio Westphal ${ }^{1}$, Aline Peluso Fezer ${ }^{1}$, Mayara Schirmer Moerschberger ${ }^{1}$ \\ and Glauco Adrieno Westphal ${ }^{1,2^{*}}$ (])
}

(c) 2022 Springer-Verlag GmbH Germany, part of Springer Nature

Dear Editor,

Although virtual communication is widely used as an alternative during the coronavirus disease 2019 (COVID19) pandemic, there are doubts about its effect on the satisfaction of family members of intensive care unit (ICU) patients $[1,2]$. We aimed to evaluate the satisfaction of family members of ICU survivors with the information for decision-making received by video call compared to face-to-face medical bulletins.

This was a case-control study conducted in a southBrazilian hospital. We excluded patients with less than $24 \mathrm{~h}$ of ICU stay, non-attendance of more than $50 \%$ of medical bulletins, and death of the patient.

Case group: medical information about the patients with COVID-19 was provided daily to relatives through video calls. Control group (non-COVID-19): face-to-face information was provided to families during or shortly after a 1-h visit. The physicians involved in communication were encouraged to adopt the best practices for news delivery [3]. Cases were matched to controls according to sex, age, date of ICU admission, Simplified Acute Physiology Score 3 (SAPS 3), use and duration of mechanical ventilation, and the need for tracheostomy.

The outcome was the satisfaction of family members with the information received for decision-making, which was assessed using the second session of the Family Satisfaction in the ICU (FS-ICU-24) questionnaire composed of two domains: "information needs" and "deliberation" $[4,5]$. The questionnaire was applied via video call.

\footnotetext{
*Correspondence: glauco.ww@gmail.com

${ }^{1}$ Centro Hospitalar Unimed, Joinville, Brazil

Full author information is available at the end of the article
}

We used Student's $t$ test or Mann-Whitney to compare continuous variables, chi-squared test for categorical variables. Multiple logistic regression model was constructed to identify predictors of an overall satisfaction assessment $>75$ (very good or excellent).

Eighty-four relatives of critically ill patients were included, 42 cases and 42 controls. Characteristics of patients and relatives are shown in Supplementary Table 1. Cases were more severe than controls according to SAPS $3(p=0.002)$. The overall satisfaction with decision-making was $>75$ in both groups, but significantly lower among cases $(75.9 \pm 16.5$ vs. $89.8 \pm 8.6$, $p<0.001)$. The satisfaction rates with the "information needs" $(p<0.001)$ and "deliberation" domains $(p<0.001)$ were lower in the case group. Eight out of nine items that compose the two domains differed significantly between groups (Fig. 1). Supplementary Table 2 shows the satisfaction ratings categorically.

After logistic regression, adjustment for confounders, belonging to the control group (OR 6.25, 95\% CI 1.77$10.85, p=0.03$ ) and honesty of information (OR 10.70, 95\% CI 2.17-15.50, $p=0.04$ ) were the variables associated with an overall satisfaction score $>75$.

Although virtual information seems to be feasible and the overall satisfaction score was good in both groups, the quality of information for decision-making was evaluated worse among family members of COVID19 patients who received information delivered online compared to face-to-face communication delivered to family members of non-COVID-19 patients. Virtual information requires great adaptability of health professionals who, in the context of the COVID-19 pandemic, 


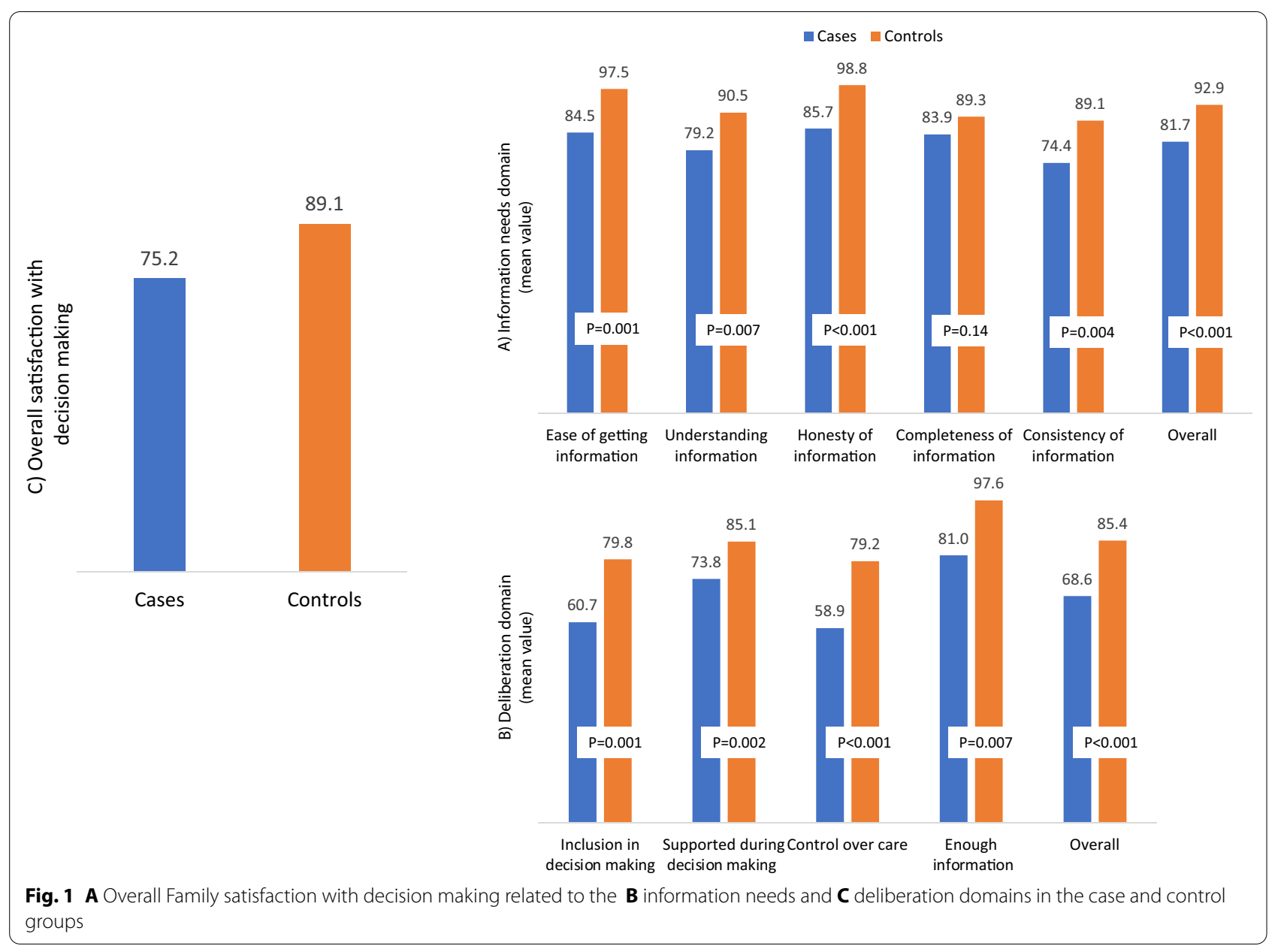

needed to develop alternatives and new skills to share information related to patients' health and to support family members in difficult decisions at a distance in the context of such a poorly known and highly lethal disease $[1,2]$. Further studies are needed to confirm our findings.

\section{Supplementary Information}

The online version contains supplementary material available at https://doi. org/10.1007/s00134-021-06616-7.

\section{Author details}

${ }^{1}$ Centro Hospitalar Unimed, Joinville, Brazil. ${ }^{2}$ Brazilian Research in Intensive Care Network (BRICNet), São Paulo, Brazil.

\section{Acknowledgements}

Special thanks to all intensivists and intensive care psychologist, and to the system analysts Lucimeri Albino and Mauricio Gonçalves for their help in the data extraction from the EHR.

\section{Declarations}

\section{Conflict of interest}

The authors declare that there are no conflicts of interest.

\section{Ethical statement}

The study was approved by the institutional Research Ethics Committee (CAAE Registration no. 29920620.7.0000.5362).

\section{Publisher's Note}

Springer Nature remains neutral with regard to jurisdictional claims in published maps and institutional affiliations.

Accepted: 27 December 2021

Published: 28 January 2022

\section{References}

1. Newcombe V, Baker T, Burnstein R, Tasker R, Menon D (2020) Clinical communication with families in the age of covid-19: a challenge for critical care teams. https://blogs.bmj.com/bmj/2020/08/11/clinical-communicat 
ion-with-families-in-the-age-of-covid-19-a-challenge-for-critical-careteams/. Accessed 15 Oct 2021

2. Back AL, Tulsky JA, Arnold RM (2020) Communication skills in the age of COVID-19. Ann Intern Med 172(11):759-760. https://doi.org/10.7326/ M20-1376

3. Baile WF, Buckman R, Lenzi R, Glober G et al (2000) SPIKES-a six-step protocol for delivering bad news: application to the patient with cancer. Oncologist 5(4):302-311. https://doi.org/10.1634/theoncologist.5-4-302

4. Wright SE, Walmsley E, Harvey SE, et al (2015) Family-reported experiences evaluation (FREE) study: a mixed-methods study to evaluate families' satisfaction with adult critical care services in the NHS. Southampton
(UK): NIHR Journals Library; December (Health Services and Delivery Research, No. 3.45.) Appendix 1, 24-item Family Satisfaction in the Intensive Care Unit questionnaire (FS-ICU-24). https://www.ncbi.nlm.nih.gov/ books/NBK333186/. Accessed 15 Oct 2021

5. Neves JL, Schwartz E, Echevarría-Guanili ME, Zanetti ACG, Heyland D, Spagnolo LML (2018) Adaptação transcultural do family satisfaction with care in the intensive care unit para o brasil/cross-cultural adaptation of the family satisfaction with care in the Intensive Care Unit for Brazil. Cienc Cuid Saude 17(4):e45069. https://doi.org/10.4025/cienccuidsaude.v17i4. 45069 